\title{
A Suitable Adaptive Illumination Compensation Method for Face Detection
}

\author{
Liu Yong-Jun*, Xie Cong-Hua \\ Department of Computer Science and Engineering,Changshu Institute of Technology, Changshu 215500,China \\ * Corresponding Author: yongjun1981@126.com
}

\begin{abstract}
In order to solve the face detection problem with complex lighting conditions, this paper presents a new method for face illumination compensation. The high-pass filtering is used to enhance edge information, the logarithmic transformation and the exponential transformtion are used to adjust the global brightness simultaneously, Nonlinear variances are applied to reduce the impact of the local highlights and shadows. The uneven lighting problem is lighten. Comparison experiments are conducted on the Yale B face dataset, Orl face dataset and our own face dataset. The results demonstrate that the illumination compensation method can significantly improve face detection rate.
\end{abstract}

Keywords : Adaboost method; face detection; illumination compensation; histogram; Retinex

\section{Introduction}

Face detection and recognition is the most complex and the most important field of pattern recognition and artificial intelligence, and can be widely used in areas such as security control, monitoring systems, and human-computer interaction. The methods based on the appearance of the face detection and identification is a classic in this field, such as average face, neural networks, support vector machines, Adaboost method [1-2]. In a non- limiting conditions, the drastic changes of the face region pixel brightness like polarized light, side light, which will lead to high light is too bright, too dark, shadow and so on and result in the detection rate .So it is essential to conduct the illumination compensation .

Currently, people often use the following three strategies to solve the lighting problem:(1) Looking insensitive to the illumination characteristics, such as a face organ stripes characterized [3] ;(2)Modeling of illumination, such as spherical harmonics modeling [4] ;
(3)Using image enhancement techniques, such as histogram equalization, the nonlinear transform [5] , Retinex [6-7]

The first method can emerge better results for the image of small illumination changes, but it is ineffective for the image of strong illumination changes; The second method is broader for application and can get better compensation effect, but the computation is complicated; The third method contra poses specific image, which can get better results, but the application has various restrictions The histogram equalization can enhance contrast, but it is prone to too bright and too dark for complex light image enhanced in the local and the uneven illumination is more uneven. The logarithmic transformation has better compensation effect of the darker facial image, but it is powerless for too bright facial images. The exponential transformation has better compensation effect for overly bright facial image, but it is powerless for too dark image. Retinex processes facial image, which will mistake to enhance the edge of the shadow. Moreover, the image processing will cost more time.

In order to solve the face detection problem with complex lighting conditions, this paper presents a new method for face illumination compensation. The high-pass filtering is used to enhance edge information, the logarithmic transformation and the exponential transformtion are used to adjust the global brightness simultaneously, and nonlinear variances are applied to reduce the impact of the local highlights and shadows. The uneven lighting problem is light-en. Comparison experiments are conducted on the Yale B face dataset, Orl face dataset and our own face dataset. The results demonstrate that the illumination compensation method can significantly improve face detection rate. Algorithm flowchart 1. 


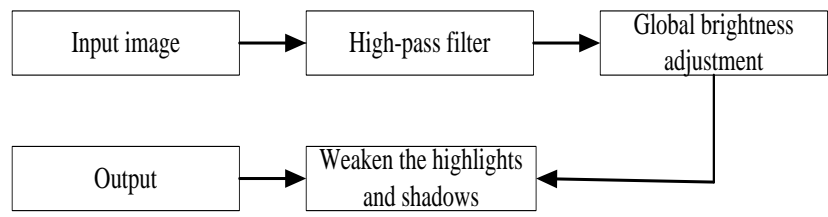

Figure 1 The method of face illumination compensation

\section{The base of Research}

Image enhancement means to enhance the useful information in the image, and its purpose is to enhance the visual effect of the image. Such as increasing the image dynamic range and improving the contrast, which can make the image clearer and the characteristics obviouser. Commonly, the methods of image enhancement are often used that include histogram equalization, the logarithmic transformation, the exponential transformation, Retinex etc.

\subsection{The histogram equalization}

The histogram equalization corrects the Original image of histogram to the gray uniform distribution histogram histogram by gray-scale transformation function, and then press histogram equalization amendment to the original image of the original image. Amending the original image depends on the balanced histogram.

The histogram equalization can enhance contrast, but it is prone to too bright and too dark for complex light image enhanced in the local and the uneven illumination is more uneven.

\subsection{The logarithmic transformation and the exponential transformtion}

(1) The logarithmic transformation: Its form is $\mathrm{g}(\mathrm{x})$ $=a \times \log x$. Its function curve is showed in Figure 2 (a), and the image is showed in Figure 2 (d) by the logarithmic transformation.

(2) The exponential transformation: Its form is $g(x)$ $=a x$. Its function curve is showed in Figure $2(\mathrm{~b})$, and the image is showed in Figure 2 (e) by the exponential transformation.

we can see from the curve of the logarithmic transformation, in $\mathrm{g}(\mathrm{x})=\mathrm{a} \times \log \mathrm{x}$, when the value of $\mathrm{x}$ is small, the growth of $\mathrm{g}$ is rapid; when the value of $\mathrm{x}$ is large, the growth of $\mathrm{g}$ is slower. Namely, the logarithmic transformation can enhance the contrast of the low

luminance region and has better illumination compensation effect on too dark images, and its shown Figure 2 (d). On the Contrary, the exponential transformation has better compensation effect for overly bright facial image, but it is powerless for too dark image and it is shown figure 2(e).

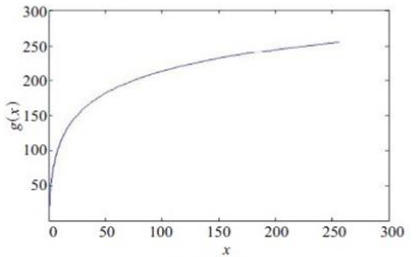

(a) Logarithmic transformation
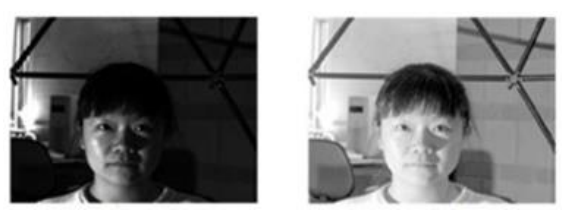

(c)Original figure (d) After the logarithmic transformation of graphics

(e) After the exponential transform the graphics

Figure 2 The index transformation function and effect

$$
\text { diagram }
$$

\subsection{Retinex}

Retinex theory that is Put forward by the Land is the perception of color constancy theory, and it's widely used in the enhancement of image, the mapping of color, the dynamic compression of color range.

In Retinex algorithm, the image is decomposed into the incident image and the reflection image.

$$
I(x, y)=R(x, y) \times L(x, y)
$$

Wherein, I represents the reflected light, that is to say, I represents the acquired image; L represents the incident light ; $\mathrm{R}$ represents the reflection properties of the object and has nothing to do with light. The Retinex algorithm essence put aside the impact of the incident light from the image I get the reflection properties of the object R.

Single scale Retinex (SSR), multi-scale Retinex (MSR) in image enhancement have some characteristics such as sharpen, color constancy, color fidelity and high dynamic range compression and so on. It can enhance image better in the absence of strong side light, but the edges of the shadows[6-7] will be enhanced in strong side light irradiation.

\section{Adaptive Face illumination compensation}




\section{1 high-pass filter}

High pass filtered by weakening the Fourier transform of the low frequency while maintaining high. Frequency is relatively constant, which makes the image becomes more clear (sharpening) [9].

After filtering, some improvement can be found, the edges of the image obtained. Therefore, this article first before logarithmic transformation of image high-pass filters Wave, in order to enhance the edges of the image information.
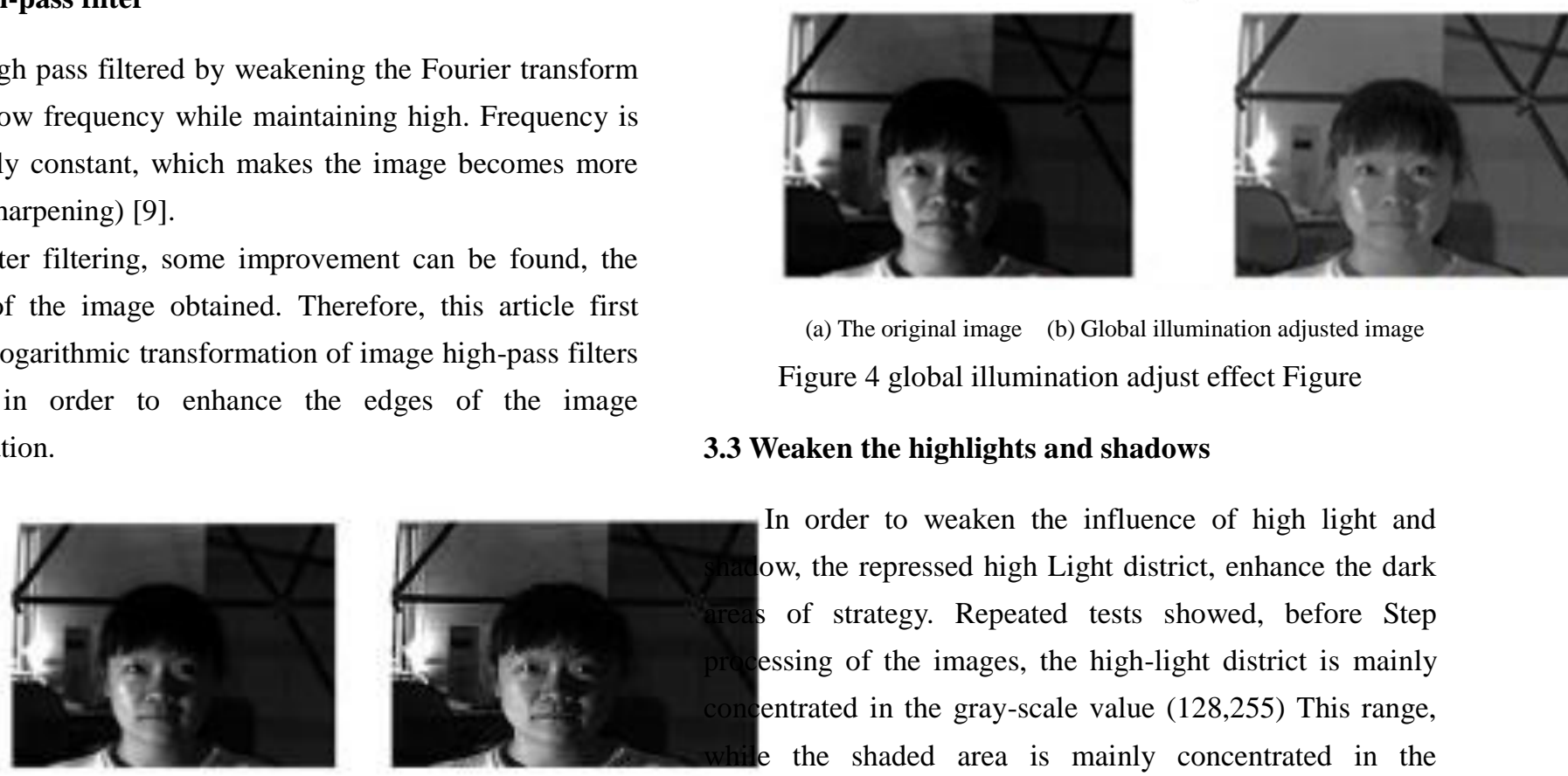

(a) The original image (b) Global illumination adjusted image

Figure 4 global illumination adjust effect Figure

\subsection{Weaken the highlights and shadows}

In order to weaken the influence of high light and ow, the repressed high Light district, enhance the dark of strategy. Repeated tests showed, before Step essing of the images, the high-light district is mainly entrated in the gray-scale value $(128,255)$ This range, the shaded area is mainly concentrated in the

$\begin{array}{ll}\text { (a) the original image } & \text { (b) filtering the image after the }\end{array}$

Figure 3 high-pass filtering effect

\subsection{Global brightness adjustment}

Biological studies have shown that the human eyes feel the brightness and enters Logarithmic relationship of a light intensity of the eye[8]. This article logarithmic transformationBasic transformation, and integration of the advantages of the exponential transformation, while improving image Low luminance and a high luminance region, to improve the image of the global illumination Love Condition. The logarithmic transformation $\ln x \mathrm{x}$ value of about 147, transform than Flat and several experiments also show that the 147 division, it is desirable Get better compensation effect. Therefore, we propose the following nonlinear transformation:

$$
g(x, y)=\left\{\begin{array}{l}
a \times \ln (f(x, y)+1), 0 \leq f(x, y) \leq 147 \\
b^{f(x, y) \times c}+d, 147<f(x, y) \leq 255
\end{array}\right.
$$

Where in, a, b, c, d for the normal number. Via the conversion of the formula (2), the image The Global Illumination situation has been greatly improved. However, for localized high Light and shadow, still powerless, as shown in Figure 4. grayscale value $(40,60)$ A range. Accordingly, it is proposed as follows piecewise nonlinear transformation:

$$
g(x, y)=\left\{\begin{array}{c}
f(x, y)+\log (1+f(x, y)), 40<f(x, y)<60 \\
a \times f(x, y)+v, 128<f(x, y) \leq 255 \\
f(x, y), \text { the } \quad \text { other }
\end{array}\right.
$$

Where, $v$ is the average luminance of the image, a high light adjustment coefficient. Figure.5 Given a different value.

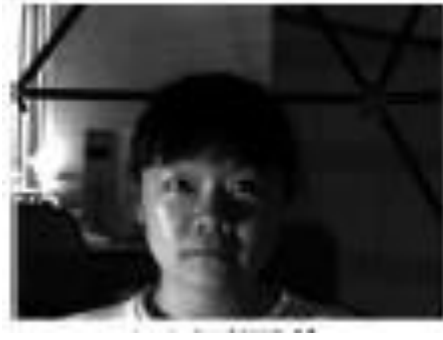

(a)the original image

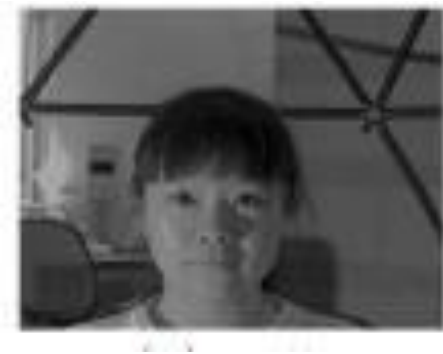

(c) $\mathrm{a}=1 / 3$

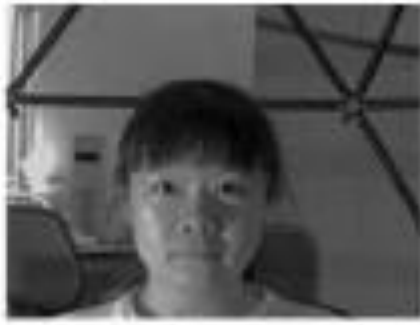

(b) $\mathrm{a}=1 / 2$

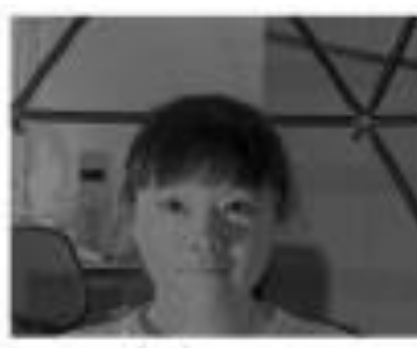

(d) $\mathrm{a}=1 / 5$
Figure 5 different values of a non-linear transformation effect diagram

As can be seen from Figure 5, when $\mathrm{a}=1 / 2$, the 
high-light areas was no significant change

Good; when $\mathrm{a}=1 / 5$, the high-light areas darken; when $\mathrm{a}=1 / 3$, the image High light and shadow to some extent weaken. Therefore, this article Take $\mathrm{A}=1 / 3$.

\section{4 algorithm processes}

(1) The initial image I (x,y).

(2)High pass filtering the image $I$, in order to strengthen the edge information obtained to the filter after the image $\mathrm{f}(\mathrm{x}, \mathrm{y})$. Laplacian high-pass filter settings is listed as follows:

$\begin{array}{ccc}- & - & - \\ 1 & 1 & 1 \\ - & 9 & - \\ 1 & & 1 \\ - & - & - \\ 1 & 1 & 1\end{array}$

(3) $f$ the implementation of the formula(3) of the transformation, fusion logarithmic transformation Exponential transform global brightness adjustment, in order to improve the image full image Bureau light situations, the image $\mathrm{g}(\mathrm{x}, \mathrm{y})$. Wherein the parameters a,b,c,d values were: $127 / \log 148,147,1$ / 255,108.

(4) $g$ perform equation (4) shown in the non-linear transformation to weaken Highlights and shadows. To obtain the final output image $\mathrm{d}(\mathrm{x}, \mathrm{y})$.

\section{Experimental results and analysis}

In order to compare different illumination compensation method for face detection shadow Ring, using OpenCV Adaboost face classifier as face detection test systems, test and uneven illumination and uniform illumination of the three people on the face database.

A test the face repository contains 10 personal nine attitude and 65 kinds of intensity of illumination of a total of 5850 images in YaleB library. This paper is light, so only selected 10650 individuals the same attitude different light face images measured again. The second experiment Orl Library, the face database contains 40 personal10 attitude and uniform illumination conditions under 400 pictures. Experiment Three face self-built library, the face database contains 20 individuals in the 200 pictures in positive light conditions.

The original image, the histogram equalization of the image after processing, Retinex image after processing of the input image as a face detection system as well as an image enhanced by this method, the comparison of each group of images face detection rate and false alarm number. Table 1, Table 2 and Table 3, respectively, are given the test results of the three sets of experiments.

Table 1 Test Results of YaleB Library

\begin{tabular}{ccc}
\hline Groups & Detection rate / (\%) & The number of false \\
& & alarms \\
\hline Artwork & 75.20 & 17 \\
Histogram equalization & 96.20 & 6 \\
Retinex (MSR) & 96.80 & 6 \\
Method & 99.80 & 11 \\
\hline Table 2 Test Results of Orl Library & \\
\hline Groups & Detection rate / (\%) & The number of false \\
\hline Artwork & & alarms \\
\hline Histogram equalization & 76.30 & 0 \\
Retinex (MSR) & 78.00 & 0 \\
Method & 80.50 & 0 \\
\hline Table 3 Test Results of Self Face Database \\
\hline Groups & Detection rate / (\%) & The number of false \\
\hline Methork & & 0 \\
\hline
\end{tabular}

From Table 1, we can see that the Retinex and this method of processing the image after the face detection rate improved to a considerable extent by the histogram equalization. Face detection rate of the proposed algorithm is the highest, but also the false alarm control in the acceptable range.

From Table 2, we can see that uniform illumination conditions, histogram equalization processing but reduced the rate of face detection, this method is still performing well.

From Table 3, we can be see that in a positive light conditions, the histogram equalization but lower detection rate, on Retinex and the method in the detection rate is improved to some extent, especially in the algorithm detection rate was the best.

Comprehensive Table 1, Table 2 and Table 3, the results can be drawn, this method can not only improve 
under uneven lighting conditions, face detection rate, also effective face detection under illumination conditions.

Table 4 shows the role of various algorithms in processing speed, the time required in the face detection. As can be seen from Table 4, the histogram equalization process followed fastest paper, but much faster than the Retinex (MSR) algorithm.

Table 4. The role of time comparing various enhancement algorithms

\begin{tabular}{cccc}
\hline Groups & \multicolumn{4}{c}{ Image size } \\
\cline { 2 - 4 } & $72 \times 84$ & $92 \times 112$ & $640 \times 480$ \\
\hline Artwork & 0.055 & 0.056 & 0.129 \\
Histogram & 0.055 & 0.059 & 0.145 \\
equalization & & & \\
Retinex (MSR) & 0.591 & 0.911 & 2.907 \\
Method & 0.058 & 0.063 & 0.343 \\
\hline
\end{tabular}

A comprehensive comparison of this method after the processing of the image, to achieve the proper balance, in terms of degree of detail-rich, contrast and overall image brightness images under uneven lighting conditions can significantly improve face detection rate, uniform illumination conditions face detection is also effective.

\section{Conclusion}

This paper presents a new image illumination compensation for face detection method. First high-pass filter to enhance edge information, while taking advantage of the logarithmic transformation and exponential transformation to adjust the overall brightness the last nonlinear changes weaken the impact of the local highlights and shadows, improve uneven image illumination, and ultimately illumination compensation. Face database, ORL face database of Yale B people as well as the self-built face database of face images under uneven illumination face image and uniform illumination experiments prove that this method can effectively light compensation, improve face detection rate.

The main purpose of this article illumination compensation in order to better detect a human face, and thus the image processing sketchy, based on face detection starting the compensation effect and cannot meet the needs of visual. Therefore, further research work can proceed from the visual effects, illumination compensation, so that it can be applied to applications such as 3D face modeling.

\section{References}

(1) Viola P, Jones M.Rapid object detection using a boosted cascade of simple features[C]//Proceedings of IEEE Conf Computer Vision and Pattern Recognition, 2001, $1: 511-518$.

(2)Phillips P J, Moon H, Syed A, et al.The FERET evaluation methodology for face-recognition algorithms [J]. IEEE Trans on Pattern Analysis and Machine Intelligence, 2000，22 (10）: 1090-1104.

(3)Miao Jun, Yin Baocai, Wang Kongqiao, et al.A hierarchi-cal multiscale and multiangle system for human face detection in a complex background using gravity-center template[J].Pattern Recognition, 1999, 32 (7) : 1237-1248.

(4)DiaoXianFeng, WangGuoYin, GongXun. A improved spherical harmonic model and in the light of the application of the face recognition [J]. Journal of ChongQing university of posts and telecommunications: Natural Science Edition, 2008, 20 (4) : 457-461.

(5)Zakaria M F, Ibrahim H, Suandi S A.A review : image compensation techniques[C]//2010 2nd International Con-ference on Computer Engineering and Technology, 2010, 7 : 404-408.

(6)RenBin. Image enhancement algorithm based on Retinex research and implementation of [D]. Nanjing: NanJing University of Science and Technology, 2009.

(7)LiuXi, LuXinHong, LiXiangLin. Based on multi-scale Retinex adaptive image enhancement method [J]. Computer application, 2009， 29 (8) : 2077-2079.

(8)JinXiaoXian, LiWeiJun, ChenXu, etc. A based on visual characteristic of bionic image enhancement algorithm [J]. Computer aided design and graphics of journal, 2010, 22 (3) : 534-537.

(9)Gonzalez. Digital image (MATLAB edition) [M]. RuanQiuQi, translation. Beijing: publishing house of electronics industry, 2005. 